\title{
Assessment of Electronic Patient Record of a super-specialty hospital
}

\author{
Prof. Rajesh Kumar Sinha \\ Department of Health Information Management Manipal College of Allied Health Sciences Manipal University, \\ Manipal - 576104 Karnataka, India.
}

\section{Dr. Smriti Rawat}

M.Sc. Hospital and Health Information Administration Manipal College of Allied Health Sciences Manipal University, Manipal.

Contact e-mail address: rajesh.sinha@manipal.edu

eHealth Sri Lanka 2010,1(suppl.1):S24

DOI: http://dx.doi.org/10.4038/sljbmi.v1i0.3569

Only the Abstract is available

\begin{abstract}
Background: Electronic Patient Record (EPR) plays a vital role in management of patient's health information. Objective: To compare the EPR in practice with Health Information Management System Society (HIMSS) standard versions 1.0 and assess the level of satisfaction among healthcare staff about existing EPR system. Methodology: The study was conducted in a super-specialty hospital in India. A checklist consisting of 30 features based on HIMSS standard was used to check the compliance of present EPR in practice. A validated and pre-tested questionnaire, based on five point Likert scale from strongly agree to strongly disagree, was used to measure the pre and post implementation EPR satisfaction among 62 healthcare professionals including doctors, nurses and administrative staff. A forum was also established to identify the problems and give necessary recommendation to resolve the issues involved in the acceptability of present EPR in practice.

Result: It has been observed that the present EPR is only compliant with 11 features out of 30 mentioned under HIMSS version 1.0. The employee satisfaction result showed that 73 per cent of doctors and nurses and 59 per cent of administrative staff were dissatisfied with the pre-implementation assessment whereas 57 per cent of doctors, 53 per cent of nurses and 59 per cent of administrative staff were found to be dissatisfied with the customisation of EPR in practice. A total of 40 per cent of doctor, 53 per cent of nurses, and 59 per cent of administrative staff felt that the core incompetency with present EPR was the present physical infrastructure. The post implementation survey result showed that 90 per cent doctors, 94 per cent nurses and 88 per cent front office staff were satisfied with the suggestions and changes in existing EPR.

Conclusion: Understanding the end user satisfaction and reinforcing them with trainings and awareness adds value and assists in continuous quality improvement, acceptability and sustainability of EPRs.
\end{abstract}

Keywords - EPR system, HIMSS, super-specialty hospital, patient's health information 